\title{
NLP EAC Recognition by Component Separation in the Eye Region
}

\author{
Ruxandra Vrânceanu, Corneliu Florea, Laura Florea and Constantin Vertan \\ The Image Processing and Analysis Laboratory (LAPI), Politehnica University of \\ Bucharest, Romania
}

\begin{abstract}
This paper investigates the recognition of the Eye Accessing Cues (EACs) used in Neuro-Linguistic Programming (NLP) and shows how computer vision techniques can be used for understanding the meaning of non-visual gaze directions. Any specific EAC is identified by the relative position of the iris within the eye bounding box, which is determined from modified versions of the classical integral projections. The eye cues are inferred via a logistic classifier from features extracted within the eye bounding box. The here proposed solution is shown to outperform in terms of detection rate other classical approaches.
\end{abstract}

Keywords: NLP; EAC; eye bounding box; eye gaze; segmentation

\section{Introduction}

The recent increase of computational capabilities has led to the development of fast and complex algorithms able to exploit new human-computer interactions. In the field of computer vision most of the research done in connection to the understanding of the functioning of human mind has been aimed at interpreting facial expressions [1] and establishing their underlying emotions [2]. Yet areas of psychology, such as the Neuro-Linguistic Programming (NLP), offer unexplored opportunities for understanding the human patterns of thinking and behavior.

NLP was introduced in the 70s by Brandler and Grinder [3], as a different model for detecting, understanding and using the patterns that appear between brain, language and body. One such model is the Eye-Accessing Cue (EAC) model that uses the positions of the iris inside the eye as an indicator of the internal thinking mechanisms of a person. The direction of gaze (Fig.1), can be used to determine the internal representational system employed by a person, who may think in visual, auditory or kinesthetic terms, and the mental activity of that person, of remembering, imagining, or having an internal dialog.

In the computer vision area, extensive research has been done in the field of detecting the direction of gaze [5]. Still most of the proposed solutions use active illumination and multiple sources of light [6]. As head mounted devices may disturb one's inner thinking mechanisms, we note that the work in [7] explores the theme only from a computer vision direction. The here-proposed solution is based on free natural light and image acquisition done with a tripodmounted camera. First, we precisely determine the eye bounding box, followed 


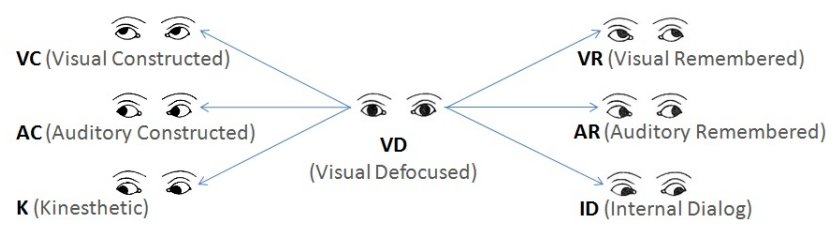

Fig. 1. 7 Classes of EACs [4]: When eyes are not used for visual tasks, their position can indicate how people are thinking (using visual, auditory or kinesthetic terms) and the mental activity they are doing (remembering, imagining, or having an internal dialog).

by a preprocessing that enhances the separation of the iris from the brow. Once the eye region is segmented, the relative position of the weighting centers of mass for the significant eye regions are processed for the EAC recognition.

Thus, in section 2 we describe the method used for isolating the eye's bounding box; inside this bounding box various methods are employed for detecting the relative iris position and the corresponding EAC, as detailed in 3 . The results achieved on a specifically constructed database are presented in section 4 and the paper ends with some conclusions.

\section{Detecting the Eye Bounding Box}

The proposed automatic solution starts with the standard Viola-Jones [8] face detection algorithm. All subsequent processing is then performed inside the face square, that is resized to $100 \times 100$ pixels. The eye bounding box is first initialized at a safe location for each eye, covering the middle-upper face quarter band (that is the lines from $y_{1}=25$ to $y_{2}=50$ ) and symmetrical vertical image quarter bands (within $x 1=20$ and $x 2=45$ for the left eye and from $x_{1}=55$ to $x_{2}=80$ for the right eye), as suggested by anthropometry and shown in Fig.2(I/II.a).

This bounding box is not sufficiently accurate and its location will be further refined. The literature comprises many approaches related to eye localization, many of them based on image integral projections [9]. The use of integral projections for the description of the eye region was further developed in [10] and we will take these works as a start base which will be improved by heuristics, designed to cover the specificity of the EAC testing (gaze variation). The precise eye bounding box detection is performed using the integral projections for the negative of the initial luminance eye regions $I$, computed as:

$$
\begin{aligned}
& P_{V}(i)=\sum_{j=x_{1}}^{x_{2}}(256-I(i, j)), \forall i=y_{1}, \ldots, y_{2} . \\
& P_{H}(j)=\sum_{i=y_{\text {top }}}^{y_{\text {bot }}}(256-I(i, j)), \forall j=x_{1}, \ldots, x_{2} .
\end{aligned}
$$



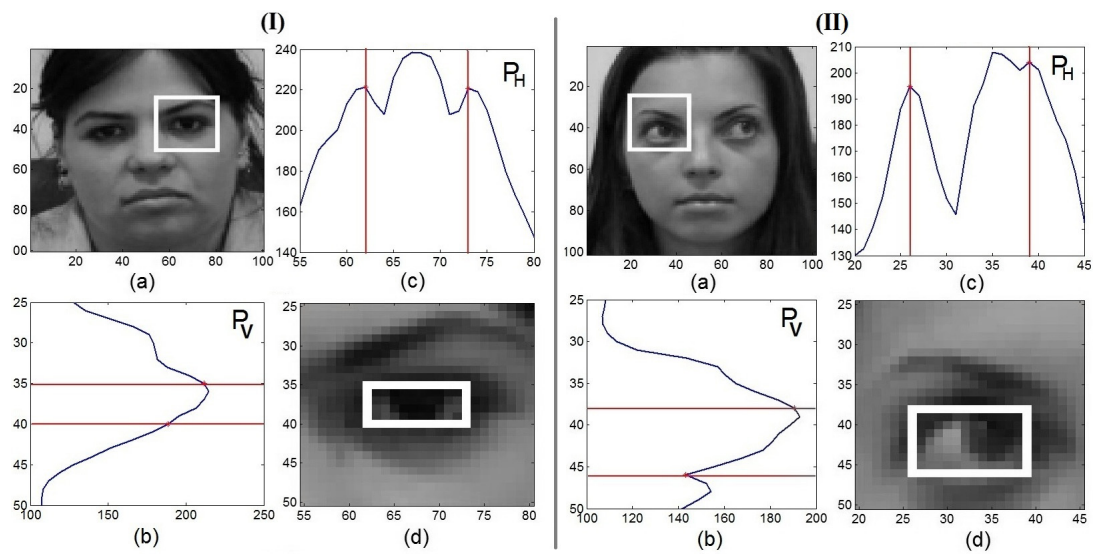

Fig. 2. Eye bounding box detections ((I)Eyes looking ahead; (II)Eyes looking sideways): a) Face square + anthropometric eye selection; b) Vertical Projection for detecting the upper/lower limits; c) Horizontal Projection for detecting the left/right limits; d) Eye Bounding Box detected inside the selection.

(a)

(b)

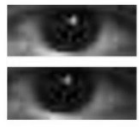

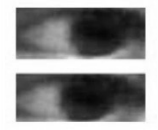

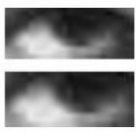

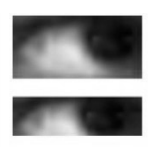
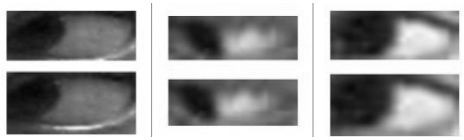

Fig. 3. Eye Bounding Box: a) Ground truth (manual markings); b) Using integral projections

First, the bounding box upper and lower limits $\left(y_{t o p}, y_{b o t}\right)$ are extracted from the vertical image projection $P_{V}$ (Fig.2(I/II.b)). Then, the lateral limits are extracted from the horizontal image projection $P_{H}$ (Fig.2(I/II.c)). The top of the bounding box is determined at 0.98 of the maximum of $P_{V}$, while the bottom is set in the first local minimum after the maximum. If there is no such a minimum (see (Fig.2(I.b)), a standard distance of 5 pixels from the maximum is used. The lateral limits are set to the local maxima, left and right, from the maximal mode in $P_{H}$. When eyes are looking sideways such maximums might not exist and the standard distance of 5 pixels is used. Visual examples of the method's performance (compared to the ground truth) are presented in Fig.3.

\section{EAC Recognition}

Once the eye bounding box has been delimited, the EAC can be determined by analyzing the positions of different eye parts. The natural choice is to analyze the position of the iris, determined with an iris center localizer inside the bounding box. For improving accuracy, we segment the regions in the eye bounding box and use their relative position and average luminance as indicators of the EAC. 


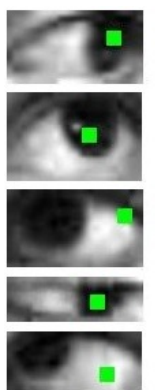

(a)

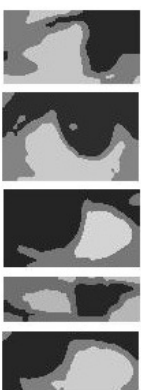

(b)

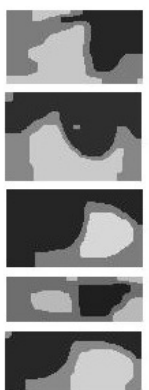

(c)

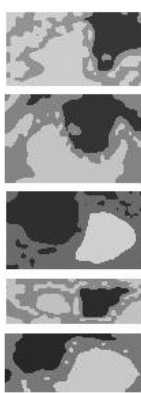

(d)

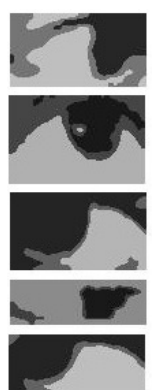

(e)

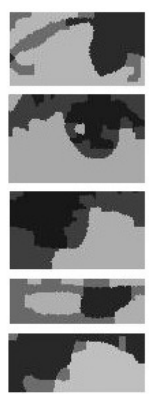

(f)

Fig. 4. Eye Features: a) Iris center using [15]; Segmentation in 3 classes using: b)KMeans; c) K-Means refined with Graph-Cuts; d) K-Means refined with pre-processing step; e) Mean Shift + region merging; f) Watershed + region merging.

Pre-processing and segmentation The segmentation is performed inside the bounding box and we experimentally determined that the optimal number of classes is 3 , corresponding generally to the iris, sclera and the surrounding skin area. Yet, as the iris and the brow tend to be spatially connected and have similar luminance values, before the actual segmentation, a pre-processing is required that separates the iris from the brow.

Starting from the observation that the eye iris is a large darker region of the eye [11], we look for the darkest, smooth neighborhood within the bounding box. This is found by selecting the areas that are darker than the median luminance value within the bounding box, in both the original image and a Gaussian lowpass smoothed image. The remaining of the eye bounding box is then lightened by a factor of 2 , such that the segmentation will generally detect the iris as a stand alone region (Fig.4(d)).

Segmentation is a well known problem and many solutions have been proposed through the years. As we do not aim at good segmentation per se, we will require a combination of good EAC recognition in a reasonable amount of time. According to the tests performed (as visually showed in Fig.4 and numerically in section 4), the best compromise is achievable using a K-means segmentation. It is possible to refine these results using Graph Cuts [12] (which imposes a smoothness constraint to reduce the number of disconnected regions and provide more compact classes (Fig.4(c)), yet the time overhead (508 msec in average for a portrait, compared to $22.5 \mathrm{msec}$ for K-means) is considerable when compared with the marginal accuracy improvement. Other tested segmentation methods were Mean Shift [13] and Watershed [14] (Fig.4(e), (f)). These methods, typically lead to over-segmentation. Yet, even though dynamic region merging is employed to consider light regions more similar, and separate the darker areas (the iris and the brow), the results under-perform the K-means method.

Post-processing and Classification To improve the region separation resulted from segmentation we rely on the same integral projections. Thus, the 
iris/sclera regions parts that were outside the minimum/maximum areas of the integral projections were cut.

Next the space given by the detected bounding box is normalized so it has a standard width (of 100 pixels), while preserving the aspect ratio. Also, since the height is variable, all eyes were aligned at the lower limit of the bounding box to ensure a better separation between eyes looking down, contained in narrow boxes, respectively up, with larger boxes.

As features describing one eye the resulting iris/sclera regions centers of mass coordinates in the normalized bounding box and average luminance are considered. To recognize the $7 \mathrm{EAC}$ classes, given the named features, various classification methods were considered. As the number of features is small, Logistic Classification [16] gives good results.

\section{Results}

Database. In order to investigate the EAC detection problem we have developed a database containing all the 7 cues. 40 subjects were asked to move their eyes according to a predefined pattern and their movements were recorded. The movements between consecutive EACs were identified, the first and last frame of each move were selected and labelled with the corresponding EAC tag and eye points were manually marked. In total, the database comprises 1170 frontal face images, grouped according to the 7 directions of gaze, with a set of 5 points marked for each eye: the iris center and 4 points delimiting the bounding box.

Training and testing procedure is the standard two-fold: half of the ground truth sets were randomly chosen to train the classifier and tune the parameters used for detecting the bounding box limits and the cue of the eyes. The testing of the algorithms (and the reported results) is done on the other half of the database.

Bounding Box Detection. As an alternative to the localization of the eye bounding box limits, one may consider the information given by a facial landmarks localization solution, such as the BoRMaN algorithm described in [17]. The algorithm iteratively improves an initial facial landmark estimate by features processed with Markov Random Fields and Support Vector Regression. To construct the necessary bounding box, we have considered the four points marking the eye, returned by the BoRMaN solution. We consider this method as being the one obviously given by the state of the art and we will show that our proposed solution outperforms it.

In order to evaluate the accuracy of the bounding box localization, inspired from the standard stringent eye center localization method [18], we have normalized the absolute error with inter-ocular distance. In evaluating the bounding box limits, only the $x$ dimension is considered for the left and the right limits, and only the $y$ dimension for the upper and lower limits. The detection error should be below 0.05. A comparative evaluation of the proposed method to the coordinates given by the solution from [17], can be seen in Fig.5 for various errors. 


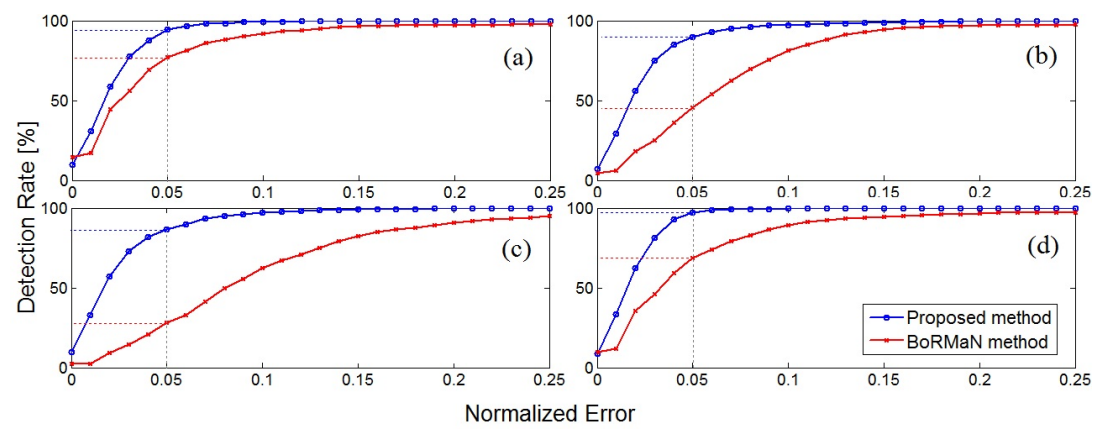

Fig. 5. Detection rate for different normalized errors (0:0.25), using BoRMaN solution (red) and the proposed solution (blue) for the bounding box limits: a) left; b) right; c)up; d)down (where the acceptable error was marked with dotted line).

Table 1. EAC Overall Recognition Rate when using: K-Means (KM), K-Means refined with Graph Cuts (GC), Mean Shift (MS), Watershed (WS)), K-Means after highlighting the darkest neighborhood (D-KM), and then refined with integral projections $($ Prj + D-KM), for bounding boxes obtained both manually and automatically(using integral projections).

\begin{tabular}{|c||c|c|c|c||c|c|}
\hline Method & KM & GC & MS & WS & D-KM & Prj + D-KM \\
\hline Manual BB & 70.90 & 71.03 & 70.35 & 71.03 & $\mathbf{7 6 . 6 8}$ & $\mathbf{8 4 . 6 3}$ \\
\hline Auto BB & 63.02 & 64.82 & 65.76 & 63.11 & $\mathbf{6 7 . 8 9}$ & $\mathbf{7 4 . 3 0}$ \\
\hline
\end{tabular}

While being more simple, the proposed solution generally gives more accurate results and provides an acceptable error for over $85 \%$ of the database.

Segmentation. Eye regions segmentation is an important step in accurate recognition of the EAC. The results obtained with various algorithms are presented in Table 1. Although the simple methods perform similarly, the K-Means segmentation gives better results, when using the pre- and post-processing steps.

$\boldsymbol{E} \boldsymbol{A} \boldsymbol{C}$ Recognition The recognition rate for each individual EAC is presented in Table 2 and the confusion matrix is shown in Fig.6(I). It can be seen that a higher confusion rate appears vertically, between eyes looking to the same side. In a NLP interpretation, this corresponds to a better separability between the internal activities and a lower one between representational systems. Visual examples of correct and false recognition are shown in Fig.6(II).

Table 2. Individual Recognition Rate for each EAC.

\begin{tabular}{|c|c|c|c|c|c|c|}
\hline VD & VR & VC & AR & AC & ID & K \\
\hline 88.32 & 76.71 & 75.00 & 61.97 & 57.86 & 63.36 & 76.81 \\
\hline
\end{tabular}




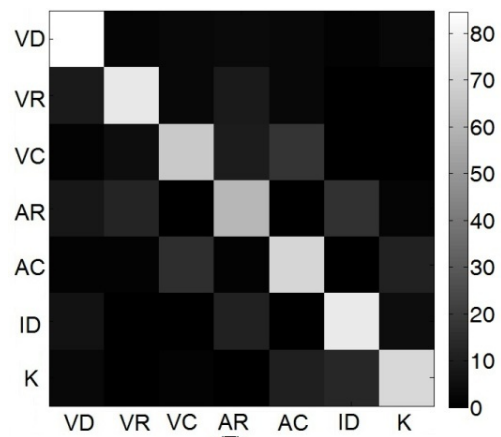

(I)
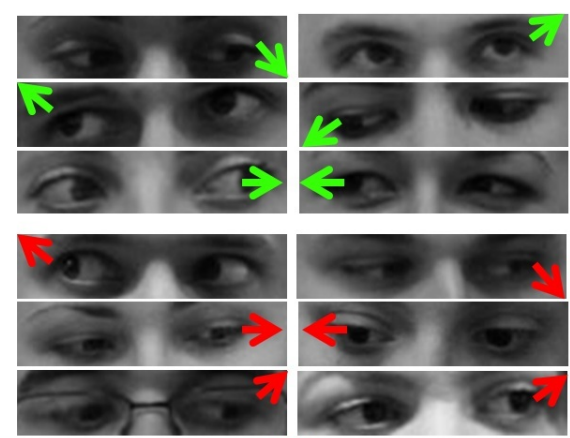

(II)

Fig. 6. (I) EAC Confusion Matrix; (II) Automatic Recognition examples: correct (green arrow) and false (red arrow).

Table 3. EAC Detection Rate for the proposed solution versus state of the art and manual markings.

\begin{tabular}{|c|c|c|c|}
\hline Method & Manual & {$[15]+[17]$} & Proposed \\
\hline DR & 84.63 & 47.82 & 74.30 \\
\hline
\end{tabular}

Comparison with related work. Given the state of the art, one intuitive way to recognize the EAC is to use the coordinates of eye fiducial points. Thus we consider as relevant the combination of the BoRMaN algorithm [17] for detecting the eye bounding box, together with the iris center, detected using the maximum isophote algorithm presented in [15]. Comparative results are presented in Table 3. As one can see, the proposed solution outperforms the state of the art, and it is close to the upper limit obtained by using the manual markings.

Although computation efficiency has not been the main focus of this paper, the proposed method is fast enough, requiring an average of $35 \mathrm{msec}$ per image, with single thread Matlab implementation with binary routines.

\section{Conclusions}

This paper has investigated new methods for recognizing the Eye Accessing Cues model, used by NLP for better understanding the mental patterns of a person. We have proven that a good recognition rate can be obtained by the joint use of segmentation and integral projection information inside the bounding box of the eye and we have proposed a solution that while is simple and fast, it outperforms existing methods.

Future research will focus on finding more features and increasing the precision of the bounding box detection, which are critical for good EAC detection. The next step will then be the EAC tracking in order to better understand the NLP patterns. The final purpose of this research is to see to which extent the 
EACs can be determined and induced for a person, and how such information could be used to improve human interaction, learning, or help in overcoming psychological traumas.

\section{Acknowledgment}

This work has been co-funded by the Sectoral Operational Program Human Resources Development (SOP HRD) 2007-2013, financed from the European Social Fund and by the Romanian Government under the contract number POSDRU/107/1.5/S/76903 and POSDRU/89/1.5/S/62557.

\section{References}

1. Fasel, B., Luettin, J.: Automatic facial expression analysis: A survey. Pattern Recognition 36(1) (1999) 256-275

2. Ekman, P.: Emotion in the Human Face. Cambridge Univ. Press (1982)

3. Bandler, R., Grinder, J.: Frogs into Princes: Neuro Linguistic Programming. Real People Press, Moab, UT (1979)

4. Fogg, A.: Nlp representational systems and eye accessing cues. www.golfhypnotist.com/nlp-representational-systems-and-eye-accessing-cues/ (2006)

5. Hansen, D., Qiang, J.: In the eye of the beholder: A survey of models for eyes and gaze. IEEE Trans. on PAMI 32(3) (2010) 478-500

6. Nakazawa, A., Nitschke, C.: Point of gaze estimation through corneal surface reflection in an active illumination environment. In: ECCV. (2012)

7. Diamantopoulos, G.: Novel eye feature extraction and tracking for non-visual eyemovement applications. PhD thesis, Univ. of Birmingham (2010)

8. Viola, P., Jones, M.: Robust real-time face detection. IJCV 57(2) (2004) 137154

9. Feng, G.C., Yuen, P.C.: Variance projection function and its application to eye detection for human face recognition. Pattern Recognition Letters 19(9) (July 1998) $899-906$

10. Zhou, Z.: Projection functions for eye detection. Pattern Recognition 37(5) (2003) 1049-1056

11. Wu, J., Zhou, Z.H.: Efficient face candidates selector for face detection. Pattern Recognition 36(5) (2003) 1175-1186

12. Y. Boykov, V.K.: An experimental comparison of min-cut/max-flow algorithms for energy minimization in vision. IEEE Trans. on PAMI 26(9) (2004) 1124-1137

13. Comaniciu, D., Meer, P.: Mean shift: A robust approach toward feature space analysis. IEEE Trans. on PAMI 24(5) (2002) 603-619

14. Meyer, F.: Topographic distance and watershed lines. Signal Processing 38 (1994) $113-125$

15. Valenti, R., Gevers, T.: Accurate eye center location and tracking using isophote curvature. In: CVPR. (2008) 1-8

16. le Cessie, S., van Houwelingen, J.: Ridge estimators in logistic regression. Applied Statistics 41(1) (1992) 191-201

17. Valstar, M., Martinez, T., Binefa, X., Pantic, M.: Facial point detection using boosted regression and graph models. In: CVPR. (2010) 2729-2736

18. Jesorsky, O., Kirchberg, K., Frischholz, R.: Robust face detection using the hausdorff distance. In: AVBPA. (2001) 90-95 